\title{
Avaliação da influência da brinquedoteca na redução da ansiedade infantil
}

\section{Evaluation of the influence of the toy to reduce child anxiety}

Evaluación de la influencia del juguete para reducir la ansiedad del niño

Larissa Evangelista Leite

ORCID: https://orcid.org/0000-0002-6971-8497 Centro Universitário Maurício de Nassau, Brasil

E-mail: larissaeleite@gmail.com

Albenice Vieira de Araujo

ORCID: https://orcid.org/0000-0003-0012-1331 Centro Universitário Maurício de Nassau, Brasil E-mail: albenicearaujo@hotmail.com

Rafaella Aparecida de Souza

ORCID: https://orcid.org/0000-0003-1750-4320

Centro Universitário Maurício de Nassau, Brasil E-mail: rafaellaasouza30@gmail.com

Beatriz Leodelgario Silva

ORCID: https://orcid.org/0000-0002-3477-4632 Centro Universitário Maurício de Nassau, Brasil E-mail: bia.silva5666@hotmail.com

Priscilla Yevelin Barros de Melo ORCID: https://orcid.org/0000-0003-2401-0898 Centro Universitário Maurício de Nassau, Brasil E-mail: yevelinpriscilla@gmail.com

Wezila Gonçalves do Nascimento ORCID: https://orcid.org/0000-0002-9295-1385 Centro Universitário Maurício de Nassau, Brasil E-mail:wezila@hotmail.com

Tácila Thamires de Melo Santos ORCID: https://orcid.org/0000-0001-7487-0605 Centro Universitário Maurício de Nassau, Brasil E-mail: tacimelotj@hotmail.com

Steffany Larissa Galdino Galisa ORCID: https://orcid.org/0000-0003-1761-2719

Universidade Estadual da Paraíba, Brasil

E-mail: steffany139@gmail.com

Raysla Maria de Sousa Almeida ORCID: https://orcid.org/0000-0001-6192-5048 Universidade Estadual da Paraíba, Brasil E-mail: almeida.rayslarms@gmail.com

Adriana Raquel Araújo Pereira ORCID: https://orcid.org/0000-0001-7815-4630 Universidade Estadual da Paraíba, Brasil E-mail: dinha_raquel@hotmail.com

Radmila Raianni Alves Ribeiro ORCID: https://orcid.org/0000-0002-8751-7437 Universidade Estadual da Paraíba, Brasil E-mail: radmilaraianni@gmail.com

Fábio Rodrigo Araújo Pereira ORCID: https://orcid.org/0000-0003-0293-1776 Universidade Federal da Paraíba, Brasil

E-mail: fabiorodrigopereira@ hotmail.com

Waleska Fernanda Souto Nóbrega ORCID: https://orcid.org/0000-0001-8140-4063 Universidade Estadual da Paraíba, Brasil E-mail: drawaleskasouto@gmail.com

Milena Edite Casé de Oliveira

ORCID: https://orcid.org/0000-0003-2266-5890 Universidade Estadual da Paraíba, Brasil E-mail: milacdo1@gmail.com

Kedma Anne Lima Gomes

ORCID: https://orcid.org/0000-0001-6720-011X Universidade Estadual da Paraíba, Brasil E-mail: kalg2010@hotmail.com 
Lorena Sofia dos Santos Andrade

ORCID: https://orcid.org/0000-0003-4766-1482 Universidade Estadual da Paraíba, Brasil E-mail: lorena_sofiacg@ hotmail.com

\begin{abstract}
Resumo
Objetivo: Ressocializar e diminuir a vulnerabilidade traumática dos demais usuários dos serviços de saúde, apresentando estratégias de grande utilidade, com uma abordagem sugestiva para a contribuição do desenvolvimento infantil, da autoconfiança, da segurança e conforto destas crianças. Metodologia: Trata-se um estudo ecológico e de revisão de literatura que teve como unidades de análise à avaliação da brinquedoteca na redução da ansiedade infantil, em especial no Brasil. Os dados são secundários obtidos via Sistema de Informação de Agravos de Notificação (SINAN). A Pesquisa foi baseada com profundidade nos estudos de casos e experiências, relatadas em 13 artigos publicados nas bases de dados Google Acadêmico e Bireme. Resultados: Verificou-se que, em todos os artigos, a relação do espaço recreativo tem uma grande influência na redução da ansiedade infantil e trata-se de um importante pilar para a recuperação e para o tratamento das crianças doentes. Considerações finais: A partir desses resultados podemos concluir que, no seguinte trabalho, a abordagem principal tem sido um estudo ao qual um leque de informações extraídas das pesquisas de dados descritos sobre a influência e a contribuição para a redução da ansiedade infantil com a implementação de brinquedotecas e jogos infantis em ambientes hospitalares, o quão tem sido proveitoso e positivo para uma boa relação entre profissional-paciente, e também terapêutico no âmbito saúdedoença.
\end{abstract}

Palavras-chave: Jogos e brinquedos; Hospitalização; Internação.

\begin{abstract}
Objective: Resocialize and reduce the traumatic vulnerability of other users of health services, presenting strategies of great utility, with a suggestive approach to the contribution of child development, self-confidence, safety and comfort to these children. Methodology: This is an ecological study and review of literature that had as units of analysis the evaluation of the toy library in the reduction of childhood anxiety, especially in Brazil. The data are secondary obtained through the Notifiable Diseases Information System (SINAN). The research was based in depth on the case studies and experiences, reported in 13 articles published in the Google Scholar and Bireme databases. Results: It was found that in all articles the relationship of the recreational space has a great influence in reducing childhood anxiety and is an important pillar for the recovery and treatment of sick children. Final considerations: From these results we can conclude that, in the following work, the main approach has been a study to which a range of information extracted from the data research described on the influence and contribution to the reduction of childhood anxiety with the implementation of playrooms and games children in hospital environments, how beneficial and positive it has been for a good relationship between professional-patient, and also therapeutic in the scope of health-disease.
\end{abstract}

Keywords: Games and toys; Hospitalization; Child.

\title{
Resumen
}

Objetivo: Resocializar y reducir la vulnerabilidad traumática de otros usuarios de los servicios de salud, presentando estrategias de gran utilidad, con un enfoque sugerente al aporte del desarrollo infantil, autoconfianza, seguridad y comodidad de estos niños. Metodología: Se trata de un estudio ecológico y revisión de la literatura que tuvo como unidades de análisis la evaluación de la ludoteca en la reducción de la ansiedad infantil, especialmente en Brasil. Los datos son secundarios obtenidos a través del Sistema de Información de Enfermedades Notificables (SINAN). La investigación se basó en profundidad en estudios de casos y experiencias, reportados en 13 artículos publicados en las bases de datos Google Scholar y Bireme. Resultados: Se encontró que, en todos los artículos, la relación del espacio recreativo tiene una gran influencia en la reducción de la ansiedad infantil y es un pilar importante para la recuperación y tratamiento de los niños enfermos. Consideraciones finales: De estos resultados podemos concluir que, en el siguiente trabajo, el abordaje principal ha sido un estudio al que se extrajo un abanico de información de la investigación de datos descritos sobre la influencia y contribución a la reducción de la ansiedad infantil con la implementación de salas de juegos. y juegos infantiles en entornos hospitalarios, lo que ha resultado beneficioso y positivo para una buena relación profesional-paciente, y también terapéutico en el ámbito de la salud-enfermedad.

Palabras clave: Juegos y juguetes; Hospitalización; Hospitalización.

\section{Introdução}

No âmbito hospitalar é contido na percepção da criança como algo desconhecido, tanto no seu aspecto físico como na rotina, e com isso, pode influenciar no comportamento infantil, mediante o seu processo saúde-doença, sendo de grande importância a busca de estratégias que ajudem na recuperação e no período de internação, para isso, a brinquedoteca entra nesse contexto como instrumento de adaptação e enfrentamento da enfermidade, onde a criança encontra no brincar a força 
para viver e permanecer no seio familiar (Costa et al., 2014). Todavia, as atividades desenvolvidas na brinquedoteca hospitalar passam por acompanhamento de profissionais como: pedagogos, psicopedagogos, psicólogos, terapeutas ocupacionais, enfermeiros, entre outros, sendo acompanhada em todo processo da brincadeira (Brito \& Perinotto, 2014).

É notório que o processo de hospitalização infantil, é algo muito marcante em suas vidas, devido demandarem sentimentos como fragilidade, impossibilidade de realizar suas atividades cotidianas, ou seja, alterando sua rotina normal como brincar e ir a escola, e brincar é algo imprescindível para a realidade da criança inclusive dentro do âmbito hospitalar, e possibilitar que auxilie na sua recuperação, é visto como algo essencial que deverá ser promovido (Costa et al., 2014; Oliveira et al., 2009).

A brinquedoteca é definida como um lugar propício para estimular crianças a brincar e se divertir, através de uma grande diversidade de brinquedos, dentro de um espaço lúdico e interativo, é possível possibilitar a criança tanto o brincar como a sua socialização, despertar a criatividade, respeito mútuo e a postura criativa e cognitiva (Costa et al., 2014). Nesta perspectiva a criança é acompanhada por uma equipe multidisciplinar, onde são avaliadas suas ações psíquicas e respostas positivas ao tratamento, através das estratégias do brincar no âmbito hospitalar.

Nessa perspectiva atual, os aspectos relacionados a hospitalizar pode não serem vistos como algo maléficos a crianças, mas hotelarias hospitalares que podem serem agradáveis e confortáveis, conduzidos a promoção da recuperação, e que do ponto de vista da lei no 11.104, de 21 de março de 2005, é obrigatório a sua instalação de brinquedoteca nos âmbitos hospitalares pediátricos, no qual consta no $2^{\circ}$ inciso da presente lei, que deverão serem promovidos de brinquedos e jogos educativos, destinados a estimular as crianças e seus acompanhantes a brincar (Brito \& Perinotto, 2014; Oliveira et al., 2009).

A internação hospitalar é considerada por muitas crianças, como sendo uma situação traumática, devido trazer diversas mudanças em sua rotina e distanciamento habitual. Com isso, a brinquedoteca entra nesse contexto, como sendo uma das estratégias que pode auxiliar na redução dos níveis de ansiedade refletidos por essas crianças como para seus familiares, que tanto aguardam atendimento como mantem-se internadas no âmbito hospitalar (Mol \& Soares, 2010).

A brinquedoteca é muito importante na vivência das crianças no âmbito hospitalar, uma vez que as atividades lúdicas desenvolvidas ajudam as crianças a manterem seus níveis de ansiedade mais controlado e melhor a sua aceitação terapêutica, conduzindo para a sua segurança e conforto, e credibilidades de seus familiares. Face ao exposto e a relevância da temática, a pesquisa justifica-se pela necessidade de buscar evidências no estudo literário e exploratório, que melhor apresente como a brinquedoteca hospitalar pode influenciar na redução da ansiedade infantil mediante processo saúde-doença, e assim subsidiar estratégias que melhor se enquadre no respeito e auxílio da assistência ofertada (Oliveira et al., 2009). Diante disso, o objetivo desse estudo é avaliar o impacto da brinquedoteca hospitalar como fator de promoção na redução da ansiedade infantil.

\section{Metodologia}

Trata-se um estudo ecológico e de revisão integrativa da literatura de abordagem quantitativa e qualitativa dos dados, que teve como unidades de analise a avaliação da brinquedoteca na redução da ansiedade infantil, em especial no Brasil. Os dados são secundários obtidos via Sistema de Informação de Agravos de Notificação (SINAN) (Pereira et al., 2018).

Foram obtidas as variáveis para a análise: Pacientes com faixa etária de 5 a 9 anos em regime de internação hospitalar em todas as regiões do Brasil, catalogados por região, entre os anos de 2014 a 2019, Os dados foram tabulados no programa Excel (Microsoft Office®, EUA, 2010) e analisados no programa Statistical Package for the Social Sciences (SPSS, IBM® company,version 24.0, EUA). Os dados foram analisados através de uma estatística descritiva com frequências absolutas e relativas (Pereira et al., 2018).

Para busca de artigos nas bases de dados realizou-se uma busca no site do PubMed (National Library of Medicine), no Site da SciELO (Scientific Eleetronic Library online), porém nesses dois sites não foram achados artigos compatíveis com a 
pesquisa. Também foi realizada busca na biblioteca de dados eletrônica da BVS (Biblioteca Virtual em Saúde), e no Google Scholar, onde nessas duas plataformas foram achados artigos compatíveis.

Os descritores foram pesquisados no site dos Descritores em Ciências da Saúde (DeCS, http://decs.bvs.br/): "Hospitalização", "Jogos e Brinquedos", combinados com o operador booleano "AND”. A pesquisa foi realizada no período de 14 de Maio de 2020 a 18 de Maio de 2020. Os artigos selecionados deveriam preencher os seguintes critérios de inclusão: Pesquisas realizadas com crianças ou acompanhantes que abordem o tema brinquedoteca nos últimos 5 anos, entre 2014 e 2019. Artigos de revisão foram excluídos. Para esta pesquisa foram utilizados os filtros: texto completo, idioma (português, inglês e espanhol) e artigos dos últimos 5 anos.

Os títulos dos trabalhos e seus devidos resumos foram analisados. Os que não permitiram a certeza de sua exclusão por meio da avaliação do resumo foram analisados na integra e categorizados conforme avaliação na Tabela Oxford Centre for Evidence-based Medicine ${ }^{\circledR}$ (Figura 1). A pesquisa nas bases de dados foi realizada de forma independente por três autores para garantir a qualidade do estudo.

Figura 1. Nível de evidência cientifica por tipo de estudo.

\begin{tabular}{|c|c|c|c|}
\hline \multicolumn{4}{|c|}{$\begin{array}{l}\text { Nivel de Evidência Científica por Tipo de Estudo - "Oxford Centre for } \\
\text { Evidence-based Medicine" - última atualizaçăo maio de } 2001\end{array}$} \\
\hline $\begin{array}{l}\text { Grau de } \\
\text { Recomendaçăo }\end{array}$ & $\begin{array}{l}\text { Nivel de } \\
\text { Evidência }\end{array}$ & $\begin{array}{l}\text { Tratamentol } \\
\text { Prevençăo - Etiologia }\end{array}$ & Diagnóstico \\
\hline \multirow{3}{*}{ A } & $1 \mathrm{~A}$ & $\begin{array}{l}\text { Revisăo Sistemática (com } \\
\text { homogeneidade) } \\
\text { de Ensaios Clínicos Controlados e } \\
\text { Randomizados }\end{array}$ & $\begin{array}{l}\text { Revisão Sistemática (com homogeneidade) } \\
\text { de Estudos Diagnósticos nível } 1 \text { Critério } \\
\text { Diagnóstico de estudos nivel 1B, em } \\
\text { diferentes centros clínicos }\end{array}$ \\
\hline & 1B & $\begin{array}{l}\text { Ensaio Clínico Controlado e } \\
\text { Randomizado com Intervalo de } \\
\text { Confiança Estreito }\end{array}$ & $\begin{array}{l}\text { Coorte validada, com bom padrão de } \\
\text { referência Critério Diagnóstico testado em um } \\
\text { único centro clínico }\end{array}$ \\
\hline & $1 \mathrm{C}$ & $\begin{array}{l}\text { Resultados Terapêuticos do tipo "tudo ou } \\
\text { nada" }\end{array}$ & $\begin{array}{l}\text { Sensibilidade e Especificidade próximas de } \\
100 \%\end{array}$ \\
\hline \multirow{5}{*}{ B } & $2 A$ & $\begin{array}{l}\text { Reviså̃o Sistemática (com } \\
\text { homogeneidade) } \\
\text { de Estudos de Coorte }\end{array}$ & $\begin{array}{l}\text { Revisão Sistemática (com homogeneidade) } \\
\text { de estudos diagnósticos de nível > } 2\end{array}$ \\
\hline & 2B & $\begin{array}{l}\text { Estudo de Coorte (incluindo Ensaio } \\
\text { Clínico } \\
\text { Randomizado de Menor Qualidade) }\end{array}$ & $\begin{array}{l}\text { Coorte Exploratória com bom padrăo de } \\
\text { Referência Critério Diagnóstico derivado ou } \\
\text { validado em amostras fragmentadas } \\
\text { ou banco de dados }\end{array}$ \\
\hline & $2 \mathrm{C}$ & $\begin{array}{l}\text { Observação de Resultados Terapêuticos } \\
\text { (outcomes research) } \\
\text { Estudo Ecológico }\end{array}$ & \\
\hline & $3 A$ & $\begin{array}{l}\text { Revisåo Sistemática (com } \\
\text { homogeneidade) } \\
\text { de Estudos Caso-Controle }\end{array}$ & $\begin{array}{l}\text { Revisão Sistemática (com homogeneidade) } \\
\text { de estudos diagnósticos de nível > 3B }\end{array}$ \\
\hline & 3B & Estudo Caso-Controle & $\begin{array}{l}\text { Seleção nắo consecutiva de casos, ou } \\
\text { padrăo de referência aplicado de forma } \\
\text { pouco consistente }\end{array}$ \\
\hline C & 4 & $\begin{array}{l}\text { Relato de Casos (incluindo Coorte ou } \\
\text { Caso-Controle de menor qualidade) }\end{array}$ & $\begin{array}{l}\text { Estudo caso-controle; ou padrão de referência } \\
\text { pobre ou não independente }\end{array}$ \\
\hline D & 5 & \multicolumn{2}{|c|}{$\begin{array}{l}\text { Opiniấo desprovida de avaliação crítica ou baseada em matérias básicas (estudo fisiológico } \\
\text { ou estudo com animais) }\end{array}$} \\
\hline
\end{tabular}


Dos 6.689 estudos identificados, 3.534 foram excluídos por não abordarem o tema proposto. Finalmente, 13 artigos atenderam aos requisitos dos critérios de inclusão e foram analisados, categorizados e interpretados. Dos artigos selecionados 11 estavam indexados na Literatura Latino Americana e do Caribe em Ciências da Saúde (LILACS), 00 no Sistema Online de Busca e Análise de Literatura Médica (MEDLINE) e 00 na Scientific Eletronic Library Online (SCIELO) e 2 Base de Dados de Enfermagem (BDENF), observe o fluxograma na (Figura 2).

O estudo encontra-se em acordo com a Resolução n 466/12 do Conselho Nacional de Saúde, que estabelece que a coleta realizada em uma base de dados de domínio público, não necessita ter a pesquisa submetida ao comitê de ética e pesquisa da instituição.

Figura 2. Fluxograma de seleção dos artigos.

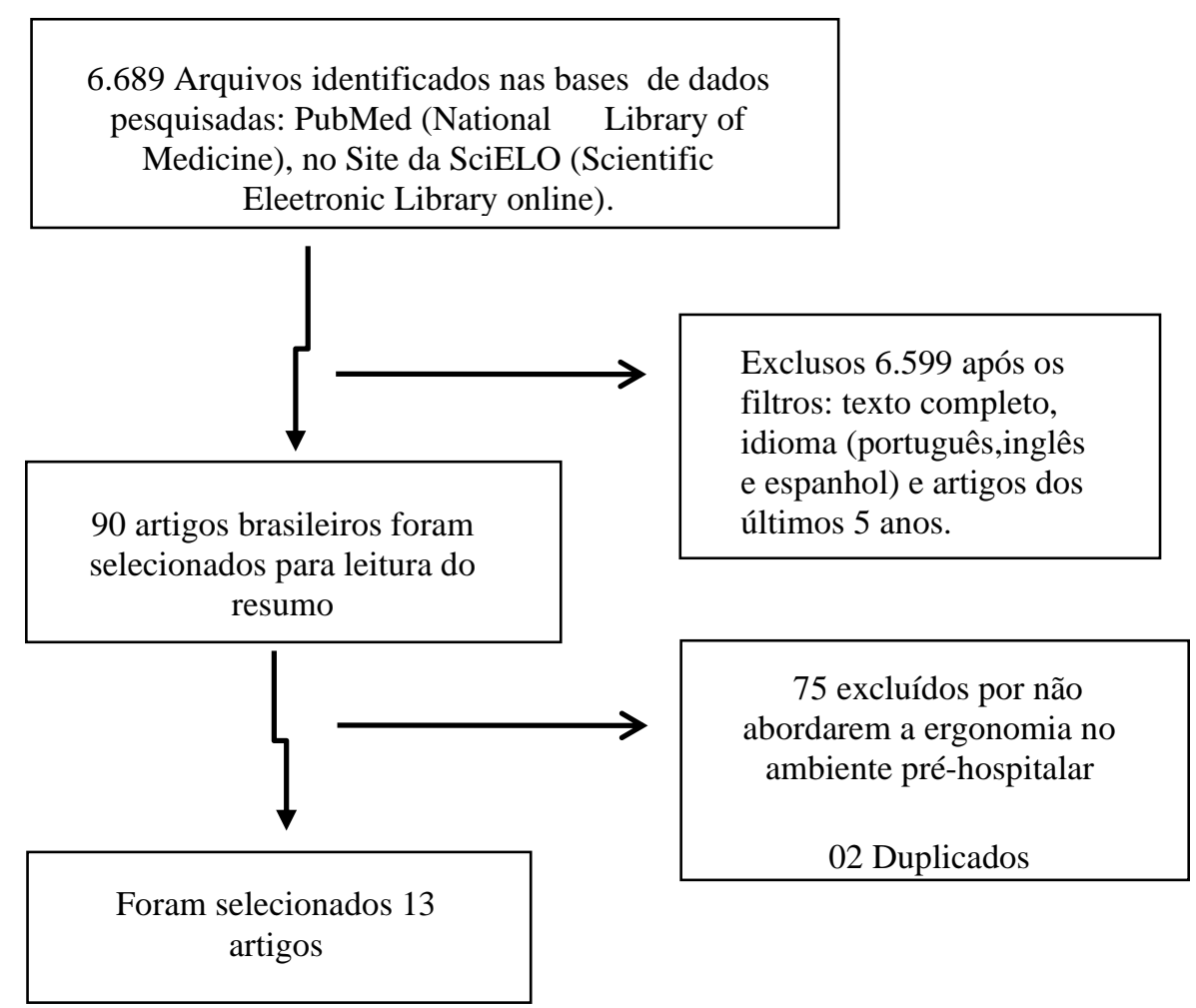

Fonte: Dados da própria pesquisa (20200.

\section{Resultados e Discussão}

No Brasil a internação na infância é muito comum na faixa etária que compreende a segunda e terceira infância (5 a 9 anos de idade). Destacando-se a região Nordeste com 1.104.794 internações para esta faixa etária e Sudeste do país com 970.410 internações nos últimos 10 anos. Um estudo de revisão que investigou as principais causas de internação na infância no Brasil identificou que as regiões com altas taxas de internações são a região Nordeste e Sudeste do país. E as principais causas de internações neste período são infecções respiratórias e parasitárias, sendo as causas comuns às pneumonias e gastroenterites que são condições sensíveis a Atenção Primária à Saúde (Pedraza \& Araújo, 2017). Há uma escassez de estudos sobre a aplicação de brinquedotecas e brinquedo terapêutico na Atenção Primária a Saúde e no ambiente hospitalar, pois a aplicação desta técnica pode contribuir com a aproximação da criança com os profissionais do serviço de saúde (Tabela 1). 
Tabela 1. Internações hospitalar em todas as regiões do Brasil, por região na faixa etária de 5 a 9 anos, entre os anos de 2014 a 2019.

\begin{tabular}{ccccccccccc}
\hline Variáveis & Norte & $\%$ & Nordeste & $\%$ & Sul & $\%$ & Sudeste & $\%$ & $\begin{array}{c}\text { Centro- } \\
\text { Oeste }\end{array}$ & \% \\
\hline $\mathbf{2 0 1 0}$ & 48.483 & 12 & 142.461 & 13 & 60.903 & 8 & 145.514 & 15 & 34.638 & 12,8 \\
$\mathbf{2 0 1 1}$ & 48.170 & 12 & 133.540 & 12 & 57.371 & 8 & 137.648 & 14 & 29.839 & 11,0 \\
$\mathbf{2 0 1 2}$ & 42.239 & 11 & 120.912 & 11 & 57.046 & 8 & 130.034 & 13 & 29.212 & 10,8 \\
$\mathbf{2 0 1 3}$ & 43.240 & 11 & 119.600 & 11 & 128.300 & 18 & 54.134 & 6 & 30.637 & 11,3 \\
$\mathbf{2 0 1 4}$ & 45.059 & 12 & 116.361 & 11 & 125.541 & 17 & 52.814 & 5 & 29.166 & 10,8 \\
$\mathbf{2 0 1 5}$ & 37.191 & 10 & 108.897 & 10 & 122.923 & 17 & 50.588 & 5 & 27.178 & 10,1 \\
$\mathbf{2 0 1 6}$ & 37.979 & 10 & 107.677 & 10 & 50.372 & 7 & 119.336 & 12 & 27.273 & 10,1 \\
$\mathbf{2 0 1 7}$ & 36.518 & 9 & 108.808 & 10 & 50.409 & 7 & 116.193 & 12 & 26.612 & 9,8 \\
$\mathbf{2 0 1 8}$ & 36.755 & 9 & 109.272 & 10 & 51.570 & 7 & 120.812 & 12 & 26.055 & 9,6 \\
$\mathbf{2 0 1 9}$ & 12.310 & 3 & 37.266 & 3 & 18.963 & 3 & 43.337 & 4 & 9.629 & 3,6 \\
\hline Total & 387.944 & 100 & 1.104 .794 & 100 & 723.398 & 100 & 970.410 & 100 & 270.239 & 100,0 \\
\hline
\end{tabular}

Fonte: Ministério da Saúde - Sistema de Informações Hospitalares do SUS (SIH/SUS)

Neste estudo, todos os artigos que se enquadram na temática e nos filtros de pesquisa propostos, foram analisados por realização de leitura dos artigos completos. Dos 13 estudos selecionados, 30,7\% dos artigos que relatam a brinquedoteca como fator significativo para redução do estresse das crianças no ambiente hospitalar, observou-se também que 38,6\% dos artigos refere-se a brinquedoteca como importante facilitador para equipe de saúde realizar os procedimentos necessários como também fonte de elo para crianças e profissionais. Outros 30,7\% artigos relatam que a brinquedoteca hospitalar além de ser elemento para humanização ao atendimento em regime de internação para as crianças, como também estimulador para redução do ócio criado no ambiente hospitalar pela mudança repentina de rotina a qual elas estavam acostumadas (Quadro 1). 
Quadro 1. Artigos selecionados na busca com autor, características metodológicas, número amostral, principais resultados, nível de evidencia e grau de recomendação.

\begin{tabular}{|c|c|c|c|c|c|}
\hline Autor (es) e ano & $\begin{array}{l}\text { Características } \\
\text { metodológicas }\end{array}$ & $\mathbf{N}$ & Principais resultados encontrados & $\begin{array}{c}\text { Nível de } \\
\text { evidencia }\end{array}$ & $\begin{array}{c}\text { Grau de } \\
\text { recomendação }\end{array}$ \\
\hline $\begin{array}{c}\text { FIORETI, } \\
\text { MANZO, } \\
\text { REGINO, } 2016 .\end{array}$ & $\begin{array}{l}\text { Pesquisa descritiva de } \\
\text { abordagem qualitativa. }\end{array}$ & 13 pais & $\begin{array}{l}\text { Minimização do estresse e melhor adaptação } \\
\text { da criança no ambiente hospitalar. }\end{array}$ & 5 & $\mathrm{D}$ \\
\hline $\begin{array}{l}\text { SOUZA et al., } \\
2015 .\end{array}$ & Estudo descritivo & 65 Mães & $\begin{array}{c}\text { Importante que tenha brinquedoteca no } \\
\text { hospital para auxiliar na recuperação e no } \\
\text { alivio do estresse gerado pelos } \\
\text { procedimentos. }\end{array}$ & 5 & $\mathrm{D}$ \\
\hline $\begin{array}{l}\text { RIBEIRO, } \\
\text { DEPIANTI, } \\
\text { MELO, } 2018 .\end{array}$ & Pesquisa qualitativa. & 08 crianças & $\begin{array}{l}\text { Obteve o resultado de que a criança durante } \\
\text { e após as brincadeiras se sentem menos } \\
\text { estressadas, se libertam do confinamento } \\
\text { hospitalar e demonstram a importância de ter } \\
\text { alguém para brincar. }\end{array}$ & 5 & $\mathrm{D}$ \\
\hline $\begin{array}{l}\text { SPOSITO et al., } \\
2018 .\end{array}$ & $\begin{array}{l}\text { Pesquisa exploratória } \\
\text { com análise qualitativa }\end{array}$ & 10 crianças. & $\begin{array}{l}\text { Na análise de dados as crianças destacaram a } \\
\text { importância de um espaço lúdico e do } \\
\text { brincar para combater a ociosidade. }\end{array}$ & 5 & $\mathrm{D}$ \\
\hline $\begin{array}{c}\text { SILVA, } \\
\text { CABRAL, } 2015 .\end{array}$ & Pesquisa qualitativa. & $\begin{array}{l}7 \text { crianças, } \\
22 \\
\text { familiares }\end{array}$ & $\begin{array}{l}\text { Após o diagnostico notou-se que houve } \\
\text { mudança no cenário e nas pessoas que } \\
\text { brincavam com as crianças, foi visto que o } \\
\text { enfermeiro precisa desenvolver essa } \\
\text { atividade de brincar. }\end{array}$ & 5 & $\mathrm{D}$ \\
\hline $\begin{array}{l}\text { SILVA et al., } \\
2018 .\end{array}$ & $\begin{array}{l}\text { Estudo qualitativo de } \\
\text { campo descritivo e } \\
\text { exploratório. }\end{array}$ & $\begin{array}{c}10 \\
\text { Familiares }\end{array}$ & $\begin{array}{l}\text { Chegaram a conclusão que os profissionais } \\
\text { da ala pediátrica desenvolvam estratégias } \\
\text { com a utilização de brinquedos terapêuticos. }\end{array}$ & 5 & $\mathrm{D}$ \\
\hline $\begin{array}{l}\text { SANTOS, et al., } \\
2014 .\end{array}$ & $\begin{array}{c}\text { Pesquisa sobre relato de } \\
\text { experiência }\end{array}$ & 03 Crianças & $\begin{array}{c}\text { Concluiu que o Modelo Lúdico mostrou uma } \\
\text { importante ferramenta para sistematizar o } \\
\text { brincar como estratégia de cuidado à criança } \\
\text { hospitalizada. }\end{array}$ & 5 & $\mathrm{D}$ \\
\hline $\begin{array}{l}\text { CALEFFI et al., } \\
2014 .\end{array}$ & $\begin{array}{l}\text { Pesquisa Convergente } \\
\text { Assistencial (PCA), de } \\
\text { abordagem qualitativa. }\end{array}$ & 07 Crianças & $\begin{array}{l}\text { Identificou que ao brincar a visão do } \\
\text { ambiente hospitalar e dos profissionais } \\
\text { torna-se menos negativa. }\end{array}$ & 5 & $\mathrm{D}$ \\
\hline $\begin{array}{l}\text { SOARES, } \\
\text { CARNEIRO, } \\
\text { BEZERRA, } \\
2014 .\end{array}$ & $\begin{array}{l}\text { Estudo descritivo com } \\
\text { abordagem qualitativa }\end{array}$ & 05 Mães & $\begin{array}{c}\text { Profissionais da ala pediátrica } \\
\text { precisam oferecer plano de cuidados lúdico } \\
\text { com estimulação do desenvolvimento } \\
\text { psicomotor, psicossocial e cognitivo. }\end{array}$ & 5 & $\mathrm{D}$ \\
\hline $\begin{array}{l}\text { SILVA et al., } \\
2015 .\end{array}$ & $\begin{array}{l}\text { Ensaio clínico } \\
\text { randomizado pareado } \\
\text { piloto. }\end{array}$ & 28 Crianças & $\begin{array}{l}\text { Em Hospitais que já existe a brinquedoteca, } \\
\text { facilita o trabalho do enfermeiro para } \\
\text { realizar procedimentos invasivos, pois as } \\
\text { crianças ficam menos ansiosas. }\end{array}$ & 1 & A \\
\hline
\end{tabular}




\begin{tabular}{|c|c|c|c|c|c|}
\hline $\begin{array}{l}\text { MISAEL, } \\
\text { FERRARI, } 2016 .\end{array}$ & $\begin{array}{l}\text { Estudo qualitativo, } \\
\text { direcionado por roteiro } \\
\text { semi-estruturado }\end{array}$ & 25 Crianças & $\begin{array}{c}\text { Observou-se que a inserção do brinquedo } \\
\text { como uma prática coadjuvante ao cuidado, } \\
\text { além } \\
\text { de favorecer assistência diferenciada, } \\
\text { humanizada e singular, é aplicável, eficaz e } \\
\text { preconizada. }\end{array}$ & 5 & $\mathrm{D}$ \\
\hline $\begin{array}{c}\text { GOMES et al., } \\
2017 .\end{array}$ & $\begin{array}{l}\text { Pesquisa descritiva, } \\
\text { exploratória de } \\
\text { abordagem quantitativa. }\end{array}$ & 32 Crianças & $\begin{array}{c}\text { O brincar minimiza o estresse da internação } \\
\text { e facilita a compreensão e aceitação dos } \\
\text { processos a serem realizados. }\end{array}$ & 5 & $\mathrm{D}$ \\
\hline $\begin{array}{c}\text { BARROSO et al., } \\
2018\end{array}$ & Pesquisa Qualitativa & - & $\begin{array}{l}\text { O brinquedo ameniza o medo e torna } \\
\text { positiva a receptividade a equipe de } \\
\text { enfermagem. Facilitando os possíveis } \\
\text { procedimentos que venham a ser realizados } \\
\text { posteriormente. }\end{array}$ & 5 & $\mathrm{D}$ \\
\hline
\end{tabular}

Fonte: Dados da própria pesquisa (2020).

Como apresenta o Quadro 1, um estudo desenvolvido no hospital de transplante com 13 pais pode identificar que após a implementação da brinquedoteca que o estresse traumático provocado pela internação foi significativamente minimizado. Para os pais os brinquedos melhoraram a adaptação das crianças ao ambiente hospitalar (Fioreti, Manzo \& Regino, 2016). Outros autores identificaram que os acompanhantes das crianças consideram a instalação de brinquedos no ambiente hospitalar uma possibilidade de auxílio na diminuição do estresse e pode ser uma ótima ferramenta na recuperação das crianças (Souza et al., 2015).

Em outra pesquisa com o objetivo de avaliar a importância do brincar, observou-se que o resultado após as brincadeiras no ambiente hospitalar foi de diminuição do estresse quando a criança tinha em sua companhia alguém partilhando com ela as brincadeiras (Ribeiro, Depianti, \& Melo, 2018). No mesmo seguimento de brincadeiras em ambiente hospitalar, observou-se que as crianças respondessem brincando através de fantoches a sua pesquisa e identificou que é necessário um ambiente lúdico para reduzir a ociosidade (Sposito et al., 2018).

Tendo o objetivo de dimensionar o espaço e as pessoas que interagem com as crianças em tratamento de câncer, as pesquisadoras notaram que houve mudança significativa das pessoas que brincavam com as crianças, passando a ter mais adultos do que crianças, visto isso entendeu-se que é necessário a equipe de enfermagem desenvolver também essa atividade lúdica para reduzir o ócio e o estresse das crianças internadas (Silva, \& Cabral, 2015). Seguindo o mesmo raciocínio dos autores já citados, foi feito um estudo com intuito de identificar a percepção dos acompanhantes a respeito das atividades lúdicas desenvolvidas no ambiente hospitalar, foi concluído que é de extrema importância os profissionais da ala pediátrica desenvolver estratégias com brinquedos terapêuticos afim de aumentar a confiança da criança, promovendo seu bem estar e para facilitar o trabalho da equipe (Silva et al., 2018).

O Serviço de Transplantes de Medula Óssea do Hospital das Clínicas da Universidade Federal do Pará, após estudo concluiu que o modelo lúdico mostrou uma importante ferramenta para sistematizar o brincar como estratégia de cuidado à criança hospitalizada, sendo esse um cuidado integral, centrado na criança e apoiado nos pressupostos da humanização (Santos et al.,2014).

Em Florianópolis, estudiosos realizaram uma pesquisa, nela verificou-se que ao brincar a visão do ambiente hospitalar e dos profissionais torna-se menos negativa, diminuindo os prejuízos de uma hospitalização mal vivenciada. E, com a utilização da brinquedoteca percebeu-se que as crianças podem vir a compreender a necessidade da internação e poder vivenciar este momento de forma mais tranquila e menos traumática (Caleffi et al., 2014). Em outra pesquisa, a equipe de 
profissionais da ala pediátrica precisa ser capaz de oferecer um plano de cuidados tendo como base a estimulação de crianças por meio lúdico, estimulando assim o desenvolvimento psicomotor, psicossocial e cognitivo (Soares, Carneiro, \& Bezerra, 2014).

Em hospitais que já existem as brinquedotecas em suas instalações foi verificado que as crianças ao utilizarem o espaço antes dos procedimentos invasivos que irão ser realizados ficam menos ansiosas em relação ao procedimento, tendo em vista que algumas precisam passar por várias punções durante sua internação, desta forma, os enfermeiros podem utilizar da brinquedoteca para facilitar o trabalho e reduzir a ansiedade das crianças (Silva et al., 2015). Observou-se que a inserção do brinquedo como uma prática coadjuvante ao cuidado, além de favorecer assistência diferenciada, humanizada e singular, é aplicável, eficaz e preconizada ao paciente pediátrico (Misael, \& Ferrari, 2016).

O estudo realizado em 32 crianças, mostrou os benefícios da técnica do brincar tanto para criança quanto para seus acompanhantes e os profissionais de saúde, minimizando o estresse decorrente do processo de hospitalização, facilitando a compreensão e aceitação dos procedimentos a serem realizados, estimulando a criança manter sua capacidade física, diminuindo a hostilidade do ambiente e proporcionando um melhor relacionamento entre a equipe de saúde e criança (Gomes et al., 2017).

Entende-se que o brinquedo é terapêutico e pode contribuir a amenizar uma relação traumática entre criança e enfermeiros, torna-se positiva a receptividade a equipe de enfermagem, facilitando os possíveis procedimentos que venham a ser realizados posteriormente (Barroso et al., 2018).

\section{Considerações Finais}

Considera-se que a implementação da brinquedoteca no âmbito hospitalar vem sendo de grande importância nas estratégias que contribuem na recuperação e no período de internação, facilitando a prestação de serviços aos pacientes, amenizando a estadia desse período um tanto quanto traumático, sendo assim podendo minimizar o estresse que já acompanha a criança diariamente em seu processo saúde-doença.

Diante desta conclusão, avaliamos os impactos destas instalações de espaços recreativos e terapêuticos nesses ambientes hospitalares, como um benéfico fator de promoção e redução da ansiedade infantil. Entretanto, apesar dos estudos selecionados mostrarem a eficácia da brinquedoteca no ambiente hospitalar, a maioria das pesquisas apresenta baixa evidência científica e o grau de recomendação é $\mathrm{D}$, por este motivo ressaltamos a necessidade de estudos experimentais sobre a temática para verificar o grau de estresse em diferentes períodos da internação hospitalar e a associação deste após a implementação de uma brinquedoteca no ambiente hospitalar.

Mesmo com um baixo grau de evidência é importante enfatizar que os estudos desenvolvidos nos hospitais e nos campos de pesquisas aqui abordados mostraram-se eficazes e significativos nas mudanças comportamentais das crianças e dos profissionais de saúde envolvidos, sendo que nos pacientes observou-se uma boa adaptação ao ambiente hospitalar e nos profissionais um melhor desenvolvimento de sua atividade de prestação de serviço e assistência, principalmente em procedimentos mais invasivos.

Os autores sugerem a importância de novos estudos sobre esta temática, visto que o número de internação infantil ainda é alto. Pode-se perceber também que há diferenças metodológicas nos estudos selecionados isso possivelmente ocorreu devido a ausência de um formulário de aplicação padronizado e as divergências quanto ao tipo de estudo e abordagem metodológica utilizada. Ressalta-se então que os procedimentos realizados durante a assistência hospitalar e a própria internação causam estresse na criança necessitando assim de uma ferramenta que proporcione abordagem assistencial qualificada e eficaz. 


\section{Referências}

Almeida, E. E. S. D. (2018). O olhar do enfermeiro sobre as práticas pedagógicas desenvolvidas no espaço da brinquedoteca hospitalar (Master's thesis).

Almeida, F. D. A., \& Sabatés, A. L. (2008). Enfermagem pediátrica: a criança, o adoslecente e sua família no hospital. In Enfermagem pediátrica: a criança, o adoslecente e sua família no hospital (pp. 421-421).

Beneduzi, A. C. (2004). Bibliotecas especiais: a biblioteca hospitalar como um repositório de saúde e bem-estar ao alcance do paciente.

Bortolin, S., \& da Silva, S. (2016). Biblioterapia no âmbito hospitalar. Informação@ Profissões, 5(1), 52-74.

Brasil. Lei n. 11.104, de 21 de março de 2005: Dispõe sobre a obrigatoriedade de instalação de brinquedotecas nas unidades de saúde que ofereçam atendimento pediátrico em regime de internação.

Brito, L. S., \& Perinotto, A. R. C. (2014). O brincar como promoção à saúde: a importância da brinquedoteca hospitalar no processo de recuperação de crianças hospitalizadas. Revista Hospitalidade, 291-315.

Caleffi, C. C. F., Rocha, P. K., Anders, J. C., Souza, A. I. J. D., Burciaga, V. B., \& Serapião, L. D. S. (2016). Contribuição do brinquedo terapêutico estruturado em um modelo de cuidado de enfermagem para crianças hospitalizadas. Revista Gaúcha de Enfermagem, 37(2).

Costa, S. A. F., Borba, C. A., \& Sanna, R. I. H. D. C. (2014). Brinquedoteca Hospitalar no Brasil: reconstruindo a história de sua criação e implantação (AU). Hist. enferm., Rev. eletronica, 206-223.

de Angelo, T. S., \& Vieira, M. R. R. (2010). Brinquedoteca hospitalar: da teoria à prática. Revista Arquivos de Ciências da Saúde, 17(2), 84-90.

de Araujo Oliveira, D. K. M., \& Oliveira, F. C. M. (2013). Benefícios da Brinquetodeca à Criança Hospitalizada: Uma Revisão de Literatura. Revista de Atenção à Saúde, 11(35).

de Carvalho, C. B. M. (2018). Biblioteca viva em hospitais: a importância da leitura como estratégia de humanização, a experiência do Instituto Fernandes Figueira. Revista Brasileira de Biblioteconomia e Documentação, 14(2), 143-154.

dos Santos, D. R., Bonfim, C. M. S., de Azevedo Mazza, V., Wall, M. L., \& das Mercês, N. N. A. (2014). Processo de brincar da criança hospitalizada guiado pelo modelo lúdico. Cogitare Enfermagem, 19(3).

Falke, A. C. S., Milbrath, V. M., \& Freitag, V. L. (2018). Estratégias Utilizadas Pelos Profissionais da Enfermagem na Abordagem à Criança Hospitalizada. Revista Contexto \& Saúde, 18(34), 9-14.

Fioreti, F. C. C. D. F., Manzo, B. F., \& Regino, A. E. F. (2016). A ludoterapia e a criança hospitalizada na perspectiva dos pais. REME rev. min. enferm, 1-6.

Fraquinello, P., Higarashi, I. H., \& Marcon, S. S. (2007). O atendimento humanizado em unidade pediátrica: percepção do acompanhante da criança. Florianópolis, 16(4), 609-16.

Lucietto, G. C., Lima, L. T. D. S., Gleriano, J. S., Justi, J., Silva, R. A. D., \& Borges, A. P. (2018). Brinquedoteca como ferramenta auxiliar no cuidado hospitalar: percepção de profissionais de enfermagem. Revista Saúde e Desenvolvimento, 12(10), 88-103.

Melo, L. D. L., \& Valle, E. R. M. D. (2010). A Brinquedoteca como possibilidade para desvelar o cotidiano da criança com câncer em tratamento ambulatorial. Revista da Escola de Enfermagem da USP, 44(2), 517-525.

Nicola, G. D. O., de Freitas, H. M. B., Gomes, G. C., Costenaro, R. G. S., Nietsche, E. A., \& Ilha, S. (2014). Cuidado lúdico à criança hospitalizada: perspectiva do familiar cuidador e equipe de enfermagem. Revista de Pesquisa Cuidado é Fundamental Online, 6(2), $703-715$.

Oliveira, L. D. B., Gabarra, L. M., Marcon, C., Silva, J. L. C., \& Macchiaverni, J. (2009). A brinquedoteca hospitalar como fator de promoção no desenvolvimento infantil: relato de experiência. Journal of Human Growth and Development, 19(2), 306-312.

Pereira, A. S., Shitsuka, D. M., Parreira, F. J., \& Shitsuka R. (2018). Metodologia da pesquisa científica. UFSM, NTE.

Santos, S. M. P. D. (2002). Brinquedoteca: o lúdico em diferentes contextos. In Brinquedoteca: o lúdico em diferentes contextos (pp. 141-141).

Silva, L. F. D., Cabral, I. E., \& Christoffel, M. M. (2010). The (im) possibilities of play for children with outpatient cancer treatment. Acta Paulista de Enfermagem, 23(3), 334-340.

Sposito, A. M. P., Santos, J. L., \& Pfeifer, L. I. (2019). Validation of the revised knox preschool play scale for the brazilian population. Occupational Therapy International, 2019.

Teixeira, S. R. D. O. Brinquedoteca hospitalar na cidade de São Paulo: exigências legais e a realidade (Doctoral dissertation, Universidade de São Paulo). 